\title{
Enhanced Mechanical Relaxation below the Glass Transition Temperature in Partially Supramolecular Networks
}

\author{
Athanasios Dimopoulos, ${ }^{\dagger}$ Jean-Luc Wietor, ${ }^{\star}$ Michael Wübbenhorst,${ }^{\S}$ Simone Napolitano, ${ }^{\S}$ \\ Rolf A. T. M. van Benthem, ${ }^{\dagger}$ Gijsbertus de With, ${ }^{\dagger}$ and Rint P. Sijbesma $* *$ \\ ${ }^{\dagger}$ Laboratory of Materials and Interface Chemistry, Department of Chemical Engineering and Chemistry, \\ Eindhoven University of Technology, Den Dolech 2, 5600 MB, Eindhoven, The Netherlands, "Laboratory of \\ Macromolecular and Organic Chemistry, Department of Chemical Engineering and Chemistry, Eindhoven \\ University of Technology, Den Dolech 2, 5600 MB, Eindhoven, The Netherlands, and ${ }^{\S}$ Laboratory for Acoustics \\ and Thermal Physics, Department of Physics and Astronomy, Katholieke University of Leuven, \\ Celestijnenlaan 200-D, B-3001, Leuven, Belgium
}

Received March 14, 2010; Revised Manuscript Received August 17, 2010

\begin{abstract}
Cross-linked polymers in which part of the covalent cross-links have been replaced by reversible cross-links have potential application in coatings with improved stress relaxation. Here we present a detailed study of materials based on copolymers of $\varepsilon$-caprolactone and L-lactic acid, containing different ratios of covalent and ureidopyrimidinone (UPy) dimer cross-links. Their thermal and mechanical properties were tested by differential scanning calorimetry (DSC), dynamic mechanical analysis (DMA), and dielectric relaxation spectroscopy (DRS). By extrapolation of the $\alpha^{*}$ relaxation process in DMA (arising from dynamics of the reversible UPy cross-links) to temperatures below the glass transition temperature $\left(T_{\mathrm{g}}\right)$, it was shown that the reversible cross-links contribute significantly to the relaxation of stress below $T_{\mathrm{g}}$ that was observed in creep measurements.
\end{abstract}

\section{Introduction}

The use of specific noncovalent interactions in polymers is a promising tool to develop responsive materials with tunable mechanical properties. In this context, the ureidopyrimidinone unit (UPy, Figure 1) is an attractive building block because it is easily synthesized on a large scale, and it reversibly forms dimers which are held together by four hydrogen bonds with a dissociation energy of $44-50 \mathrm{~kJ} / \mathrm{mol}$ in chloroform and toluene, respectively. ${ }^{1}$ These values are in between the strength of single hydrogen bonds and covalent bonds, ${ }^{2}$ which makes the interaction reversible, yet strong. Because dimerization is a reversible, dynamic process, the UPy unit has been used in the preparation of self-assembled supramolecular systems, ${ }^{3}$ in supramolecular polymer networks ${ }^{4,5}$ and for biomedical applications. ${ }^{6,7} \mathrm{UPy}$ dissociation/association allows for fast stress relaxation at elevated temperatures for materials designed to display shapememory properties. ${ }^{8,9}$ The dynamic properties of the UPy group also offer opportunities for slow stress relaxation at lower temperatures, a process that is important in reducing stresses that accumulate during application of coatings on metal substrates due to differences in thermal expansion coefficients.

Recently, we reported on the properties of polyesterpolyurethane thermosets containing different fractions of noncovalent UPy cross-links in addition to covalent cross-links at constant cross-link density. The materials with supramolecular cross-links were shown to have enhanced stress relaxation at temperatures above, as well as below the glass transition temperature. ${ }^{10}$ Dissociation of UPy dimers in the networks was shown to give rise to an additional relaxation mechanism, resulting in the presence of two distinct rubber plateaus in the elastic modulus

\footnotetext{
*Corresponding author. E-mail: r.p.sijbesma@tue.nl. Telephone: +31402473111 .
}

measured by dynamic mechanical analysis (DMA). The UPycontaining materials also displayed enhanced creep and stress relaxation below $T_{\mathrm{g}}$.

Here, we investigate the mechanical relaxation mechanisms of these materials in detail (Scheme 1). One of the most important issues that was not fully addressed in the initial paper concerned the question to what extent the UPy-related relaxation process that was observed at $45^{\circ} \mathrm{C}$ above $T_{\mathrm{g}}$ in DMA contributes to stress relaxation under operating conditions of coatings, typically $10-20{ }^{\circ} \mathrm{C}$ below $T_{\mathrm{g}}$. Answering this question requires an extensive analysis of the temperature dependence of the relaxation processes connected to UPy dynamics. To this end, films with different contents of UPy groups were investigated by DMA using temperature scans at multiple frequencies in order to determine the thermal activation parameters of this relaxation process. We compare these results with relaxation times over a larger time window obtained by broadband dielectric relaxation spectroscopy (DRS). Finally, we relate these results to stress relaxation behavior of the materials using a simple mechanical model.

\section{Experimental Section}

Details of chemicals, synthesis and characterization have been published previously. ${ }^{10}$

Differential Scanning Calorimetry (DSC). Thermal properties $\left(T_{\mathrm{g}}\right)$ of the free-standing films were investigated with differential scanning calorimetry on a TA Instruments Q2000 differential scanning calorimeter with an RCS cooling unit under a nitrogen atmosphere with heating and cooling rates of $10 \mathrm{~K} \cdot \mathrm{min}^{-1}$ (samples of 8-12 $\mathrm{mg}$ were measured). The temperature range was from -20 to $+120{ }^{\circ} \mathrm{C}$.

Dynamic Mechanical Analysis (DMA). The mechanical properties of the fully covalent material and the UPy containing ones were tested in tensile mode on a TA Instruments Q800 series 
DMA with a gas cooling accessory (GCA) under nitrogen atmosphere. The dimensions of the specimens tested were $12 \mathrm{~mm} \times 6 \mathrm{~mm} \times 0.5 \mathrm{~mm}$. The dynamic tests for the determination of $T_{\mathrm{g}}$ and the cross-link density (XLD) were performed at a heating rate of $3 \mathrm{~K} \cdot \mathrm{min}^{-1}$ and a frequency of $1 \mathrm{~Hz}$. The experiments at multiple frequencies were performed in isothermal steps of $5 \mathrm{~K}$. The temperature range was from 0 to $140{ }^{\circ} \mathrm{C}$ and the frequency range $0.1-21.5 \mathrm{~Hz}$. The creep measurements were performed at different temperatures $\left(5 \mathrm{~K}\right.$ below the $T_{\mathrm{g}}$ of each UPy containing material and $25 \mathrm{~K}$ above the $T_{\mathrm{g}}$, respectively) at a stress of $0.5 \mathrm{MPa}$ and a creep time of $200 \mathrm{~min}$, followed by a recovery time of $200 \mathrm{~min}$. Each measurement was performed on two identical specimens to test the reproducibility. In all cases a preload force of $0.01 \mathrm{~N}$ was applied.

Dielectric Relaxation Spectroscopy (DRS). The dielectric relaxation spectra of all material compositions were measured by means of a high precision dielectric analyzer (Alpha analyzer, Novocontrol Technologies) combined with a Quatro temperature controller and a nitrogen-flushed Cryosystem. Temperature dependent experiments were performed by consecutive isothermal frequency sweeps in the range of $10^{-1}-10^{7} \mathrm{~Hz}$ and in the temperature range from -140 to $+100{ }^{\circ} \mathrm{C}$ in steps of $5 \mathrm{~K}$, which resulted in an effective heating rate of about 0.5 $\mathrm{K} \cdot \mathrm{min}^{-1}$. Circular samples for the dielectric measurements were prepared from thin sheets of a typical thickness of $0.3 \pm 0.03 \mathrm{~mm}$

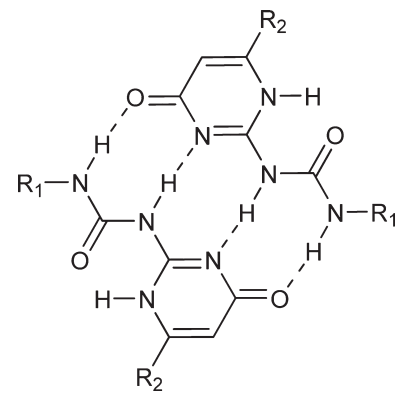

Figure 1. Ureidopyrimidinone (UPy) dimer. (diameter $21 \mathrm{~mm}$ ) and were inserted between the rigid electrode systems of the dielectric sample cell that ensured a well-defined sample geometry during the experiment.

\section{Results and Discussion}

The polyester urethanes used in this work were described in detail in our previous report. These materials are networks formed from branched, random copolymers of $\varepsilon$-caprolactone (CL) and L-lactic acid (LLA) with a monomer ratio CL/LLA of 20:80 (Scheme 1). The three-arm branched precursor polymers were partially end-functionalized with UPy groups and further cross-linked with Desmodur N3400, a nonvolatile, commercial diisocyanate. We refer to the cured films as $\mathbf{F}-\boldsymbol{u}$, with $u$ the percentage of UPy-cross-links; e.g. F-30 refers to the triol prefunctionalized with $30 \%$ UPy-hexamethyleneisocyanate, the remaining $70 \%$ of the initial hydroxyl groups being cross-linked with Desmodur N3400.

Thermal Properties by DSC and Mechanical Properties by DMA. The thermal properties of the materials were characterized using DSC and the mechanical properties of the films (F-0 to $\mathbf{F - 4 0})$ were studied by DMA in order to determine the glass transition temperature, $T_{\mathrm{g}}$, and the corresponding cross-link densities $\left(v_{\mathrm{e}}\right)$ calculated using eq $1^{11}$

$$
E^{\prime}=3 v_{e} R T\left(T \gg T_{\mathrm{g}}\right)
$$

where $E^{\prime}$ is the storage modulus measured under tension, $v_{e}$ is the calculated cross-link density, $R$ is the universal gas constant, and $T$ is the absolute temperature. As evident from Table $1, T_{\mathrm{g}}$ increases slightly with increasing amount of UPygroups. In view of elucidating the role of thermo-reversible cross-links, this effect might complicate a direct comparison between the different materials. However, for application in coatings $T_{\mathrm{g}}$ is a parameter that may be adjusted by tuning the CL:LLA monomer ratio.

Multifrequency DMA Measurements. DMA measurements at frequencies ranging from 0.1 to $21.5 \mathrm{~Hz}$. were

Scheme 1. (a) Cartoon with Topology of the Cross-Linked Network with Covalent and Non-Covalent Cross-Links; (b) Chemical Structure of Monomeric Units Provided with a UPy Non-Covalent Cross-Linking Unit and the Covalent Cross-Linker Desmodur N3400

a)

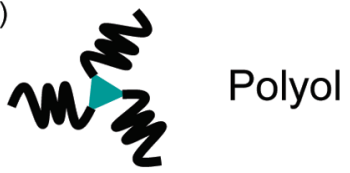

N Covalent cross-link

- Supramolecular cross-link

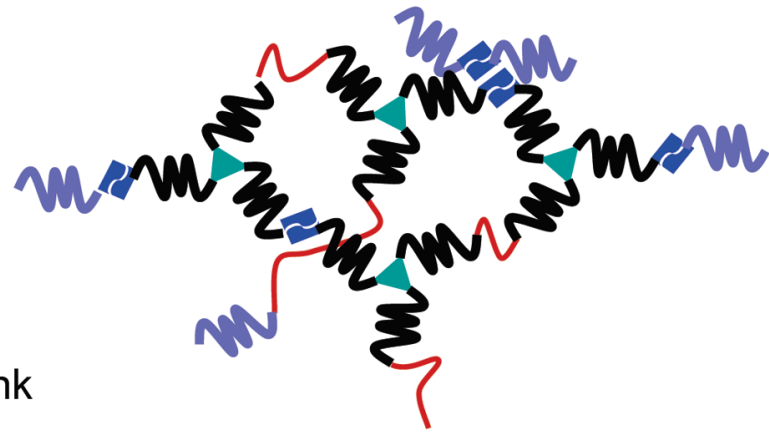

b)
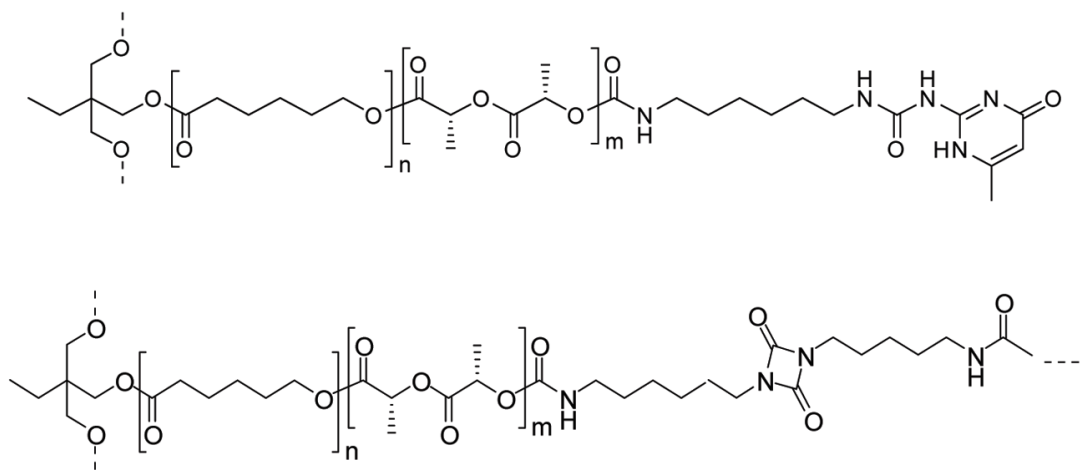
Table 1. Summary of Properties of the Polyester-Urethane Networks

\begin{tabular}{lccccc}
\hline coating & $u(\%)$ & $T_{\mathrm{g}}\left({ }^{\circ} \mathrm{C}\right) \mathrm{DSC} / \mathrm{DMA}^{a, b}$ & $\alpha^{*}$ relaxation DMA $\left({ }^{\circ} \mathrm{C}\right)^{b}$ & ${\text { storage modulus } E^{\prime}(\mathrm{MPa})^{c}}_{\mathrm{cross}^{c} \text { link density }\left(\mathrm{mol} \cdot \mathrm{cm}^{-3}\right)}$ \\
\hline F-0 & 0 & $30 / 35$ & & $6.95\left(110^{\circ} \mathrm{C}\right)$ & $727 \times 10^{-6}$ \\
F-10 & 10 & $33 / 38$ & 85 & $4.42\left(113^{\circ} \mathrm{C}\right)$ & $455 \times 10^{-6}$ \\
F-20 & 20 & $35 / 42$ & 87 & $4.09\left(117^{\circ} \mathrm{C}\right)$ & $421 \times 10^{-6}$ \\
F-30 & 30 & $36 / 42$ & 87 & $3.65\left(117^{\circ} \mathrm{C}\right)$ & $376 \times 10^{-6}$ \\
F-40 & 40 & $37 / 44$ & 88 & $2.16\left(119^{\circ} \mathrm{C}\right)$ & $220 \times 10^{-6}$
\end{tabular}

${ }^{a}$ DSC measurements were performed at a heating rate of $10 \mathrm{~K} \mathrm{~min}^{-1} .{ }^{b}$ Maximum of loss modulus $E^{\prime \prime}$ at a heating rate of $3 \mathrm{~K}$ min ${ }^{-1}$ and a frequency of $1 \mathrm{~Hz} .{ }^{c}$ Storage modulus measured $75 \mathrm{~K}$ above $T_{\mathrm{g}}$ and respective temperature (indicated in brackets). These values were used for the calculation of cross-link densities.
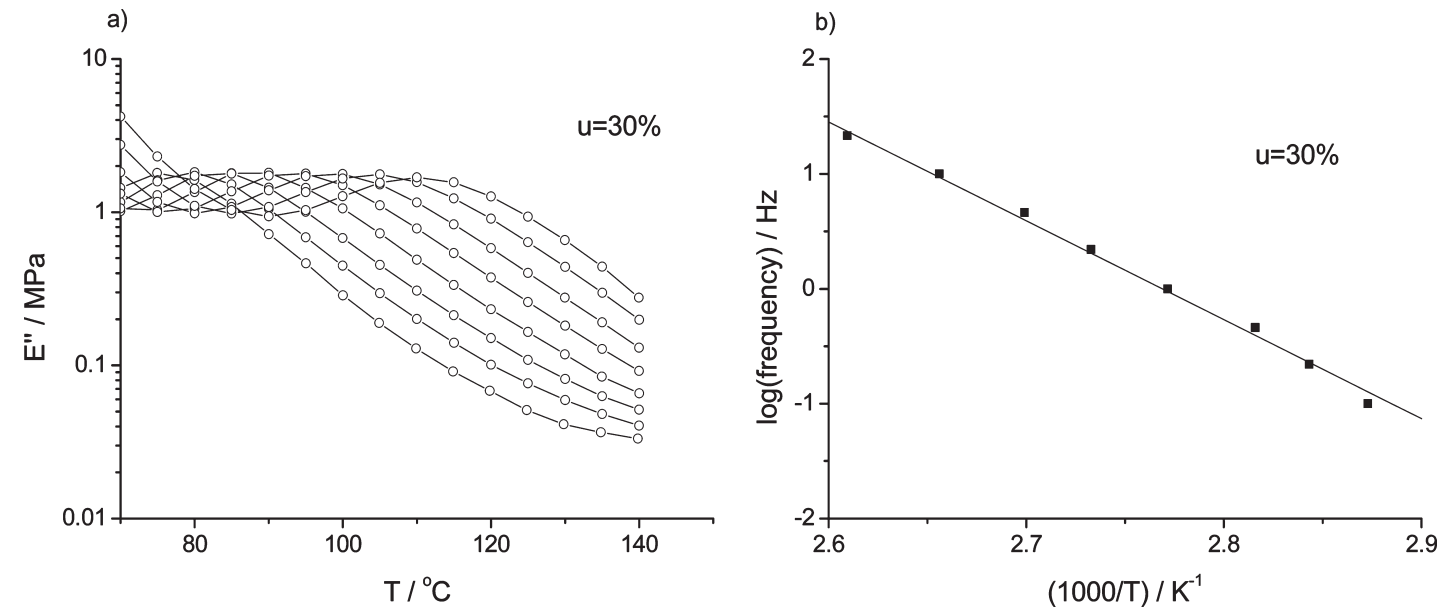

Figure 2. (a) Isochronal representation of the mechanical loss modulus $E^{\prime \prime}$ for the $\mathbf{F}-\mathbf{3 0}$ material in the region of the high-temperature relaxation for different frequencies: $0.1,0.22,0.46,1,2.2,4.6,10$, and $21.5 \mathrm{~Hz}$. (b) Arrhenius plot of the peak relaxation frequency $f_{\alpha}{ }^{*}$ for the F-30 material.
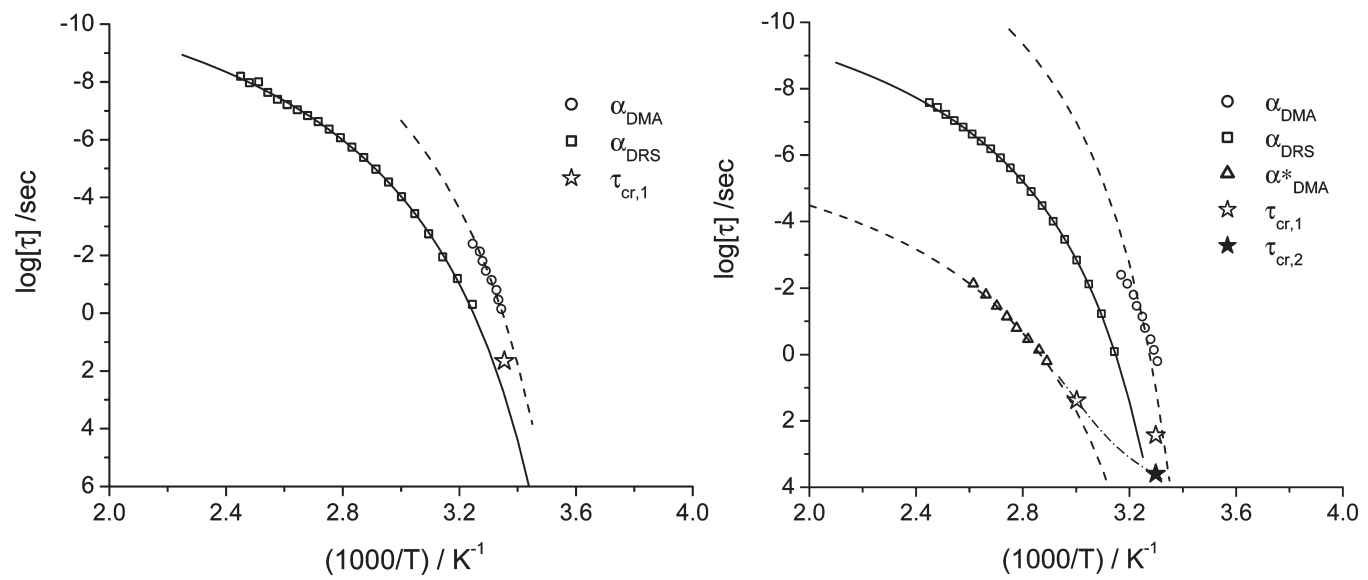

Figure 3. Relaxation times of the glass transition $(\alpha)$ and the high temperature relaxation process $\left(\alpha^{*}\right)$ obtained from mechanical and dielectric spectroscopy: (a) F-0 material and (b) F-20 material. The stars indicate relaxation times obtained from creep experiments at $T_{\mathrm{g}}-5 \mathrm{~K}$ and $T_{\mathrm{g}}+25 \mathrm{~K}$, the dot-dash line illustrates a possible scenario as discussed below in the text.

performed for the materials $\mathbf{F - 0}$ to $\mathbf{F - 4 0}$. The first maximum in the loss modulus, $E^{\prime \prime}$ as a function of temperature (Figure 2a) is associated with the glass transition and will be referred to as the $\alpha$-relaxation (not shown), while the second maximum in the loss modulus (shown in Figure 2a) is related to the dynamics of dissociation/association of the hydrogen bonding unit and will be referred to as the $\alpha^{*}$-relaxation. The temperature dependence of the latter processes is plotted in an Arrhenius representation in Figure 2b. The curvature in the plot is typical for dynamic processes in polymers that are linked to the glass transition. Accordingly, the apparent activation energy of about $170 \mathrm{~kJ} / \mathrm{mol}$, derived from the average slope of the plot is substantially higher than the activation energy observed for dissociation of UPy dimers in solution. ${ }^{1}$ Calculating the apparent activation energy from the Vogel-Fulcher-Tammann (VFT) equation yields a similar value.

Broadband DRS Measurements. To corroborate the mechanical relaxation data, dielectric relaxation spectroscopy (DRS) was performed for all materials in the frequency range of $10^{-1}-10^{7} \mathrm{~Hz}$ and temperature range from -140 to $+100{ }^{\circ} \mathrm{C}$. Some essential results from the DRS experiments are given in the Arrhenius representation (Figure 3) that summarizes the relaxation times $\tau_{\alpha \text { DRS }}$ for the $\alpha$-process together with the mechanical relaxation times for the materials $\mathbf{F - 0}$ and $\mathbf{F - 2 0}$. A more rigorous analysis and interpretation of the dielectric relaxation data will be subject of a separate publication. From Figure 3, we conclude that for all materials, the glass transition is manifested by a corresponding dielectric and the mechanical relaxation process $\left(\alpha_{\text {DRS }}\right.$ and 
a)
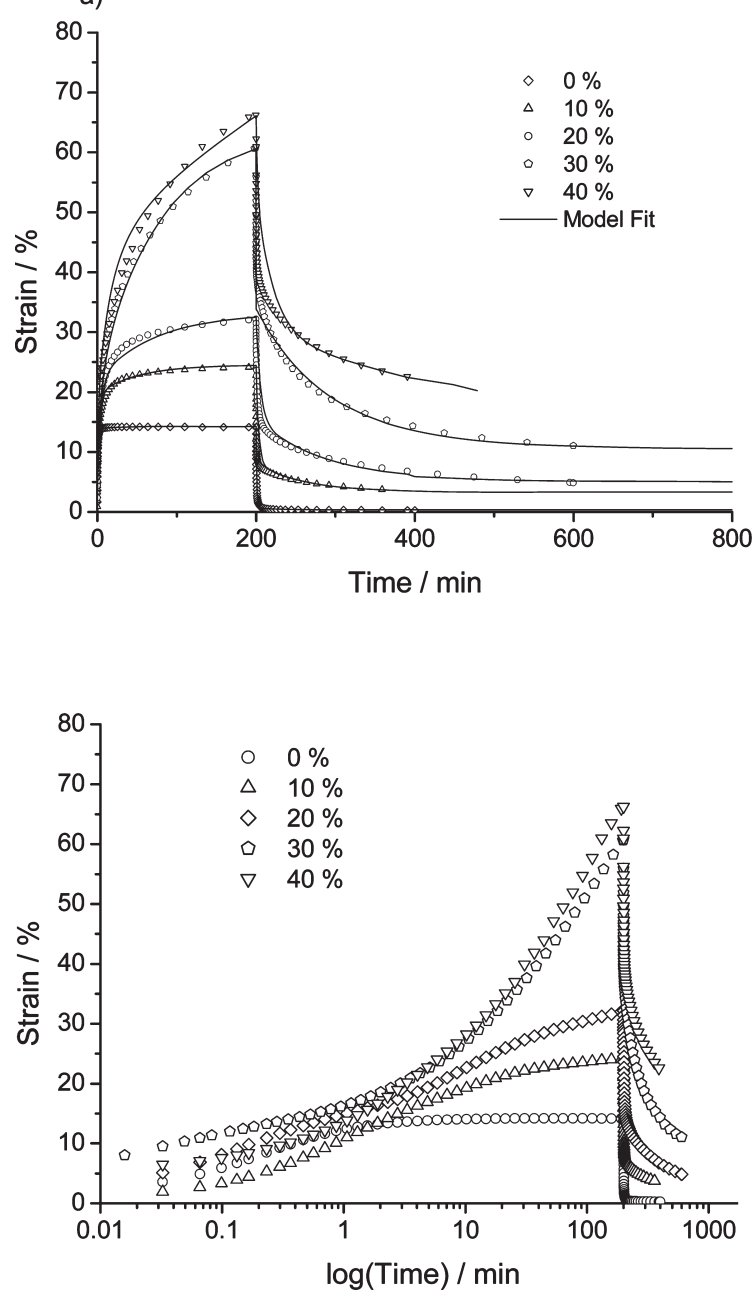
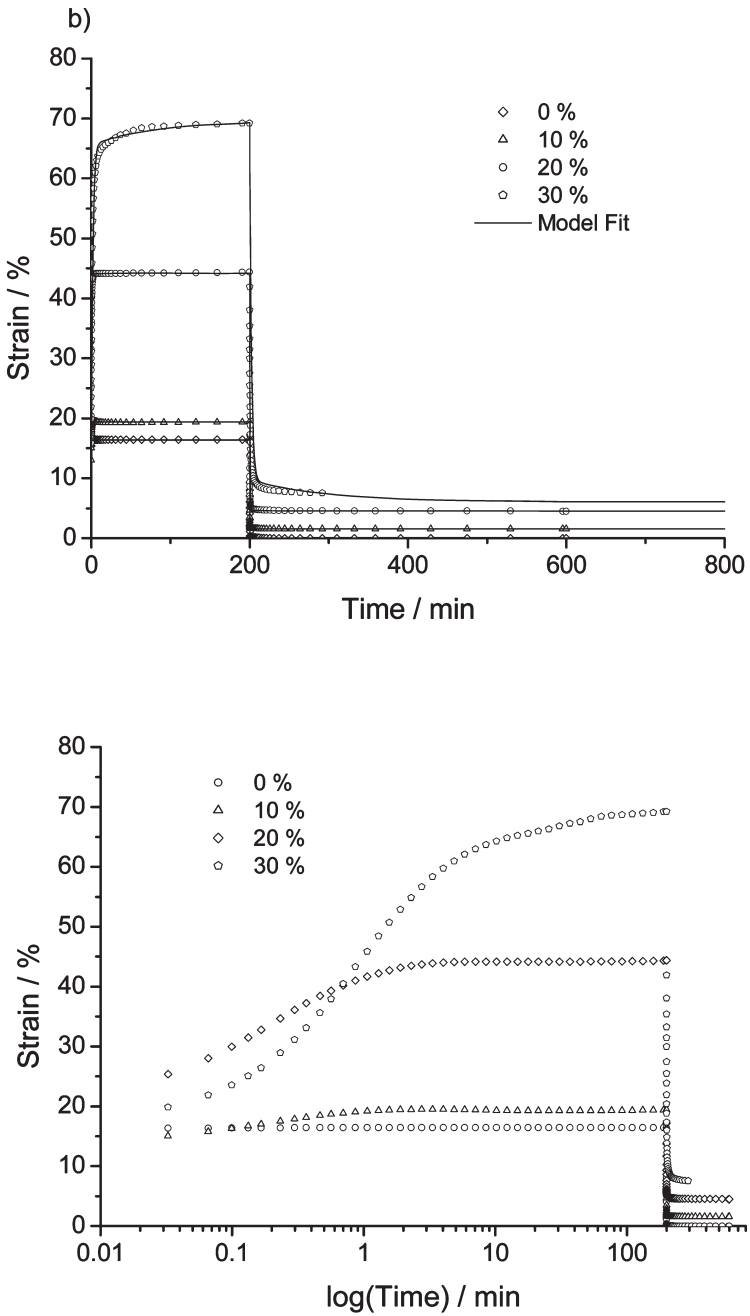

Figure 4. Creep and strain recovery measurements at a stress of $0.5 \mathrm{MPa}$ (symbols) and fit results (lines) at (a) $5 \mathrm{~K}$ below the glass transition temperature and (b) $25 \mathrm{~K}$ above the glass transition temperature of films $\mathbf{F - 0}$ to $\mathbf{F 4 0}$. For more clarity the data are given on both linear and logarithmic time scales.

$\left.\alpha_{\text {DMA }}\right)$. Although the mechanical and dielectric relaxation times are systematically shifted with respect to each other by $2-3$ decades, they should obey the same temperature dependence according to the VFT, eq $2^{12}$

$$
\tau(T)=\tau_{\infty} \exp \left[\frac{E_{v}}{R\left(T-T_{v}\right)}\right]
$$

where $\tau$ and $\tau_{\infty}$ denote the relaxation times, $T_{\mathrm{v}}$ is the Vogel (reference) temperature, and $E_{\mathrm{v}}$ is the apparent activation energy in the high temperature limit. Applying eq 2 to the dielectric relaxation time data yields excellent fits of the experimental data over a wide frequency range as seen in Figure 3 (solid lines). By shifting the VFT-fit curve vertically, i.e., using the same VFT-parameters $E_{\mathrm{V}}$ and $T_{\mathrm{V}}$ and solely adjusting the pre-exponential factor $\tau_{\infty}$, we can further check the two mechanical relaxation processes easily for their temperature dependence with respect to the dielectric relaxation times. The result is again displayed in Figure 3 indicating a surprisingly good fit for both the mechanical $\alpha$-process and the slow mechanical $\alpha^{*}$-relaxation.

Comparing the graphs of the DMA and DRS relaxation times reveals that the peaks in the relaxation spectra cover several decades in frequency. Since the gap between the DMA and DRS peak relaxation times (2-3 decades) is smaller than the width of the individual relaxation time spectra ( $\sim 8$ decades $)$, we can state that there is significant overlap between mechanical and dielectric relaxation time data. While a common temperature dependence for the dielectric and mechanical $\alpha$-process is generally expected, the strict coupling between the $\alpha^{*}$ - and the $\alpha$-dynamics implies that the dynamics of the UPy network are driven by the segmental mobility, in line with earlier work on UPybased supramolecular polymers. ${ }^{13}$ Assigning the mechanical $\alpha^{*}$-process to the dynamics of the UPy network is also supported by the fact that this relaxation process is not observed for the F-0 material (Figure 3a).

Creep/Strain Recovery Experiments by DMA. In order to relate the mechanical and dielectric relaxation processes to the materials behavior at larger deformations we have performed creep and strain recovery experiments at a temperature just below the calorimetric $T_{\mathrm{g}}$ as well as at $T=T_{\mathrm{g}}+$ $25 \mathrm{~K}$ (cf. Figure 4). Strains up to $70 \%$ were measured, which were established to be proportional to stress up to at least $1 \mathrm{MPa}$. The data of experiments at $0.5 \mathrm{MPa}$ stress were analyzed using a model consisting of a Maxwell and three Kelvin-Voigt elements in series. ${ }^{14}$ Attempts to fit the combined creep and strain recovery traces with less (1 or 2) Kelvin-Voigt elements did not yield satisfying results while the use of more than 3 elements resulted in large statistical errors of the estimated parameters. The dashpot in the Maxwell element was utilized mainly for the measurements 
Table 2. Model Parameters for Creep and Strain Recovery Measurements at 0.5 MPa Stress at 5 K below the Glass Transition Temperature of Films F-0 to F-40

\begin{tabular}{|c|c|c|c|c|c|c|c|c|c|}
\hline coating & $u(\%)$ & $E_{\mathrm{o}}(\mathrm{MPa})$ & $E_{1}(\mathrm{MPa})$ & $\log \left(\tau_{1} / \mathrm{s}\right)$ & $E_{2}(\mathrm{MPa})$ & $\log \left(\tau_{2} / \mathrm{s}\right)$ & $E_{3}(\mathrm{MPa})$ & $\log \left(\tau_{3} / \mathrm{s}\right)$ & $\log \left(\tau_{D M A}\right)^{a}$ \\
\hline F-0 & 0 & 1.47 & 0.037 & 1.65 & 1.85 & 3.08 & 5.56 & 3.38 & 0.19 \\
\hline F-10 & 10 & 0.15 & 0.031 & 2.19 & 0.10 & 3.48 & 5.01 & 3.56 & 0.13 \\
\hline F-20 & 20 & 0.10 & 0.029 & 2.43 & 0.07 & 3.59 & 0.13 & 3.76 & 1.00 \\
\hline F-30 & 30 & 0.05 & 0.044 & 2.56 & 0.02 & 3.59 & 0.03 & 3.78 & 0.50 \\
\hline$F-40$ & 40 & 0.07 & 0.039 & 1.97 & 0.02 & 3.02 & 0.01 & 4.38 & -0.40 \\
\hline
\end{tabular}

${ }^{a}$ Extrapolated relaxation times based on a constrained VFT-fit of the DMA data, cf. Figure 3.

Table 3. Model Parameters for Creep and Strain Recovery Measurements at 0.5 MPa Stress at $25 \mathrm{~K}$ above the Glass Transition Temperature of Films F-0 to F-30 ${ }^{a}$

\begin{tabular}{|c|c|c|c|c|c|c|c|c|c|c|}
\hline coating & $u(\%)$ & $E_{\mathrm{o}}(\mathrm{MPa})$ & $E_{1}(\mathrm{MPa})$ & $\log \left(\tau_{1} / \mathrm{s}\right)$ & $E_{2}(\mathrm{MPa})$ & $\log \left(\tau_{2} / \mathrm{s}\right)$ & $E_{3}(\mathrm{MPa})$ & $\log \left(\tau_{3} / \mathrm{s}\right)$ & $\eta(\mathrm{MPa} \cdot \mathrm{s})$ & $\log \left(\tau_{D M A} / \mathrm{s}\right)^{b}$ \\
\hline F-0 & 0 & 16.7 & 0.031 & -0.22 & 500 & 3.38 & 14.9 & 3.56 & 0.088 & \\
\hline F-10 & 10 & 0.321 & 0.028 & 0.78 & 250 & 3.41 & 16.5 & 3.59 & 0.168 & 1.59 \\
\hline F-20 & 20 & 0.109 & 0.013 & 1.38 & 125 & 3.48 & 7.4 & 3.68 & 0.211 & 1.73 \\
\hline F-30 & 30 & 0.082 & 0.008 & 2.04 & 0.124 & 3.68 & 90.3 & 3.82 & 0.343 & 1.59 \\
\hline
\end{tabular}

${ }^{a}$ The F-40 film ruptured at a creep time of $\sim 100 \mathrm{~min} .{ }^{b}$ Extrapolated relaxation times based on a constrained VFT-fit of the DMA data, cf. Figure 3.

above $T_{\mathrm{g}}$ in order to account for irreversible creep. The relaxation times as well as the viscosity (corresponding to the extra dashpot) are summarized in Figure 3 and Tables 2 and 3 , respectively.

Figure 4 displays the time dependent strain at constant stress (creep) and subsequent strain recovery for the five sample compositions. As expected, the fully covalent network shows almost fully elastic behavior indicated by a fast response $\left(<1 \mathrm{~s}\right.$ at $T_{\mathrm{g}}+25 \mathrm{~K} ;<1 \mathrm{~min}$ at $\left.T_{\mathrm{g}}-5 \mathrm{~K}\right)$ and nearly full strain recovery. With increasing UPy content, the time response reveals an additional slow retardation and relaxation process, a larger ultimate strain value due to the decrease of the ultimate network modulus, and an increasingly incomplete strain recovery, i.e. irreversible creep.

In order to compare the creep/recovery relaxation times $\tau_{\mathrm{cr}}$ (see Tables 2 and 3) with the $\tau$-values from the DMA measurements, we have added the $\tau_{\mathrm{cr}}$-data to the Arrhenius presentation given in Figure 3 as well as in the Supporting Information. To consider only the most significant contributions from our fit model formally assuming three creep relaxation times (3 Kelvin-Voigt elements) we have selected the relaxation times associated with the lowest modulus $E_{x}$. It is evident from Table 3 that $\tau_{1}$ is the only dominant contribution to the relaxation behavior at $T_{\mathrm{g}}+25 \mathrm{~K}$ while the experiments at $T_{\mathrm{g}}-5 \mathrm{~K}$ (cf. Table 2 ) need the consideration of two characteristic times $\left(\tau_{1}\right.$ and $\left.\tau_{2}\right)$.

From Figure 3, we can see that the creep relaxation times $\tau_{\mathrm{cr}, 1}$ match very well the mechanical relaxation times from DMA within typically 1 order of magnitude. It is obvious that in the polymer melt $\left(T_{\mathrm{g}}+25 \mathrm{~K}\right)$ the creep experiment (relaxation time $\tau_{\mathrm{cr}, 1}$ ) captures the UPy dynamics, while at lower temperatures, the $\tau_{\mathrm{cr}, 1}$ time corresponds to the time scale of the segmental dynamics. However, the slow retardation/relaxation times, $\tau_{\mathrm{cr}, 2}$, obtained from the lowtemperature creep experiments do neither fit the extrapolated DMA relaxation times for the $\alpha$ nor those for the $\alpha^{*}$-relaxation. On the other hand, inspection of the time-dependent strain data in Figure 4 (left graphs) clearly confirm that this slow $\tau_{\mathrm{cr}, 2^{-}}$ dynamics must be associated with the (almost) full relaxation of the UPy network on the time scale of the creep-experiment (200 min) as implied by the high strain plateau that compares well with the rubber plateau at high temperatures. In other words, the stress experiments unambiguously reveal an accelerated dynamics of the UPy network at temperature close to the calorimetric glass transition temperature.

In order to rationalize this faster dynamics we have to realize that at the glass transition temperature, the segmental dynamics, i.e., the $\alpha$-process, get frozen while the system passes from an equilibrium state (melt) to a nonequilibrium glass. This transition typically occurs when the structural relaxation time is in the order of $100-1000 \mathrm{~s}$, depending on the actual cooling rate. On the other hand, there is a continuous rearrangement of the UPy-groups, the "UPydynamics", which is driven by the segmental dynamics (same temperature dependence) but proceeding at a much longer time scale- a time that actually corresponds to the "lifetime" of a given configuration of the $\mathrm{H}$-bonding network. At $T_{\mathrm{g}}$, however, the UPy dynamics would be in the order of $10^{6}$ $10^{7} \mathrm{~s}$, about 4 decades slower than the $\alpha$-process, provided that the system had the chance to equilibrate. We think that the latter issue is the reason for the faster UPy dynamics as revealed by the creep experiments: Upon cooling the polymer melt, the UPy dynamics starts to freeze already above $T_{\mathrm{g}}$ (about $T_{\mathrm{g}}+20 \mathrm{~K}$; see suggested trajectory in Figure 3), since the UPy rearrangement process cannot equilibrate any longer because of its huge relaxation time. As a consequence, the UPy network gets arrested in an out of equilibrium state, in which the H-bonding groups can sample only a restricted number of accessible local configurations, leading to faster dynamics and an (eventually) less effective physical network.

The enhanced dynamics of UPy relaxation is supported by the analogy to the structural relaxation of polymers in the glassy state (physical aging) from which it is known that the temperature dependence of the structural relaxation time becomes flatter below $T_{\mathrm{g}}$, i.e., the activation energy of the remaining modes of the structural relaxation in the glassy state does not obey the VFT-law any longer and turns to an Arrhenius behavior. ${ }^{15}$

\section{Conclusions}

We have demonstrated that extrapolation of the $\alpha^{*}$-process in DMA (arising from dynamics of the reversible UPy cross-links) results in relaxation times in good agreement with those obtained by fitting of creep measurements well above the glass transition temperature. In contrast to this, we have shown that at $5 \mathrm{~K}$ below the calorimetric glass transition temperature, the UPy-related relaxation process identified by creep and strain recovery experiments is significantly faster than predicted from extrapolation of the $\alpha^{*}$-process to this temperature. In fact, the UPy relaxation unexpectedly is fast enough to facilitate stress relaxation on the time scale of hours to days, while on the other hand, it is slow enough to maintain sufficient hardness on shorter time scales. The measurements also demonstrate an increased overall relaxation amplitude of the materials under stress with increasing amounts of UPy cross-links. These combined effects of introducing 
UPy groups in the materials show that replacing part of the covalent cross-links in a network with thermoreversible hydrogen bonds is a promising concept for application in industrial coatings since the UPy's offer an additional relaxation mode (at reasonable speed and amplitude considering the coating's lifetime and typical service temperature) on top of the already well-known creep mechanisms. Hence, strong hydrogen bonds offer an additional engineering parameter for pre-emptive stress relaxation while maintaining a favorable balance of hardness versus ductility.

Acknowledgment. The authors thank the Dutch IOP Self Healing Materials (project SHM0626), SupraPolix BV, and DSM Engineering Plastics for their financial support. S.N. acknowledges FWO (Fonds Wetenschappelijk OnderzoekVlaanderen) for a postdoctoral scholarship. Jan Leys is acknowledged for performing the dielectric spectroscopy experiments.

Supporting Information Available: Figures showing DSC traces of F-0 to F-40, VFT fits of DRS data of films F-10, F-30 and $\mathbf{F - 4 0 , ~ C r e e p ~ a n d ~ s t r a i n ~ r e c o v e r y ~ d a t a ~ o f ~ f i l m s ~} \mathbf{F - 0}$ to $\mathbf{F}-\mathbf{4 0}$ and modeling using Burger's ${ }^{16}$ and combined Kelvin-Maxwell models and tables of VFT activation energies and relaxation times and creep and strain recovery parameters (Burger model) of films F-0 to F-40. This material is available free of charge via the Internet at http://pubs.acs.org.

\section{References and Notes}

(1) Söntjens, S. H. M.; Sijbesma, R. P.; van Genderen, M. H. P.; Meijer, E. W. J. Am. Chem. Soc. 2000, 122, 7487-7493.

(2) Beijer, F. H.; Sijbesma, R. P.; Kooijman, H.; Spek, A. L.; Meijer, E. W. J. Am. Chem. Soc. 1998, 120, 6761-6769.
(3) Sijbesma, R. P.; Beijer, F. H.; Brunsveld, L.; Folmer, B. J. B.; Hirschberg, J. H. K. K.; Lange, R. F. M.; Lowe, J. K. L.; Meijer, E. W. Science 1997, 278, 1601-1604.

(4) Lange, R. F. M.; van Gurp, M.; Meijer, E. W. J. Polym. Sci., Part A: Polym. Chem. 1999, 37, 3657-3670.

(5) Brunsveld, L.; Folmer, B. J. B.; Meijer, E. W.; Sijbesma, R. P. Chem. Rev. 2001, 101, 4071-4097.

(6) Dankers, P. Y. W.; Adams, P. J. H. M.; Löwik, D. W. P. M.; van Hest, J. C. M.; Meijer, E. W. Eur. J. Org. Chem. 2007, 3622-3632.

(7) Dankers, P. Y. W.; van Leeuwen, E. N. M.; van Gemert, G. M. L.; Spiering, A. J. H.; Harmsen, M. C.; Brouwer, L. A.; Janssen, H. M.; Bosman, A. W.; van Luyn, M. J. A.; Meijer, E. W. Biomaterials 2006, 27, 5490-5501.

(8) Li, J.; Viveros, J. A.; Wrue, M. H.; Anthamatten, M. Adv. Mater. 2007, 19, 2851-2855.

(9) Lendlein, A.; Kelch, S. Angew. Chem., Int. Ed. 2002, 41, 2034-2057.

(10) Wietor, J.-L.; Dimopoulos, A.; Govaert, L. E.; van Benthem, R. A. T. M.; With, G. de; Sijbesma, R. P. Macromolecules 2009, 42, 6640 6646.

(11) Treloar, L. R. G. The Physics of Rubber Elasticity, 2nd ed.; Oxford University Press (Clarendon): London, 1958.

(12) van Turnhout, J.; Wübbenhorst, M. J. Non-Cryst. Solids 2002, 305, $50-58$.

(13) Wübbenhorst, M.; Folmer, B. J. B.; van Turnhout, J.; Sijbesma, R. P.; Meijer, E. W. IEEE Trans. Dielectr. Electr. Insul. 2001, 8, 365-372.

(14) Ward, I. M.; Handley, D. W. An Introduction to the Mechanical Properties of Solid Polymers; John Wiley \& Sons: Chichester, U.K., 1993.

(15) Cangialosi, D.; Wübbenhorst, M.; Groenewold, J.; Mendes, E.; Schut, H.; van Veen, A.; Picken, S. J. Phys. Rev. B 2004, 70, 224213.

(16) Burgers, J. M. Mechanical considerations-model-systems-phenomenological theories of relaxation and of viscosity. In First report on Viscosity and Plasticity; Burgers, J. M., Ed.; Nordemann Publishing Company: New York, 1935. 